American Journal of Biochemistry and Biotechnology 2 (2): 61-65, 2006

ISSN 1553-3468

(C) 2005 Science Publications

\title{
Nanocrystalline Powders of Alkaline-Earth Phosphates as Precursors for Bioceramics
}

\author{
${ }^{1,2}$ Parhi. Purnendu, A. Ramanan, ${ }^{2}$ Alok R. Ray ${ }^{1}$ \\ ${ }^{1}$ Centre for Biomedical Engineering, Indian Institute of Technology, Delhi, India \\ ${ }^{2}$ Department of Chemistry, Indian Institute of Technology, Delhi, India
}

\begin{abstract}
Bone is a nanocomposite made of calcium phosphates and collagen. Collagen has a typical fibrous structure, with diameter ranging from $100 \mathrm{~nm}$ to $2 \mu \mathrm{m}$. It is suggested that calcium hydroxyapatite, $\mathrm{Ca}_{10}\left(\mathrm{PO}_{4}\right)_{6}(\mathrm{OH})_{2}$ in the size range 5-50 nm embedded in the collagen framework provides mechanical strength to bone. Among calcium phosphates, apatites are found to be the most suitable for bone regeneration due to its biocompatibility. A contemporary theme is to prepare nanocrystallites of alkaline-earth phosphates that can be employed as precursors for making novel bioceramics. In this paper, we present our attempts to prepare nano-sized particles of alkaline-earth hydroxyapatites, $\mathrm{A}_{10}\left(\mathrm{PO}_{4}\right)_{6}(\mathrm{OH})_{2}$ where $\mathrm{A}=\mathrm{Ca}, \mathrm{Sr}$ and $\mathrm{Ba}$ through a metathetical route. Our work involved the use of same reactants treated under different reaction conditions. While hydrothermal route yielded well-crystalline nanorods, microwave resulted in agglomerated hydroxyapatites and reverse micellear route gave low crystalline apatites with less agglomeration.
\end{abstract}

Key words: Alkaline-earth hydroxyapatite, metathesis, nanorods

\section{INTRODUCTION}

Human bone is a wonder material made by nature and is a true nanocomposite. It is a complex and a highly specialized form of connective tissue pertaining to the formation of the skeleton of the body. Bone, not only provides mechanical support but also elegantly serves as a reservoir for minerals, particularly calcium phosphate. It is a good example of a dynamic tissue, since it has a unique capability of selfregenerating or self-remodeling to a certain extent throughout the life without leaving a scar ${ }^{[1]}$. The bone mineral is mainly composed of hydroxyapatite (HAp) and the bone protein is predominantly from collagen $^{[2,3]}$. Here, collagen acts as a structural framework in which plate-like tiny crystals of HAp are embedded to strengthen the bone ${ }^{[4]}$. The bone collagen has a typical fibrous structure, with diameter ranging from $100 \mathrm{~nm}$ to $2 \mu \mathrm{m}$. Similarly, HAp in the bone mineral is mostly in the form of nanocrystals, with dimensions of about 5 to $50 \mathrm{~nm}{ }^{[5,6]}$. Bone can exhibit different types of integration between organic and inorganic materials, leading to significant variations in their mechanical properties. The ratio of both the components reflects the compromise between toughness (high inorganic content) and fracture strength (low inorganic content). All attempts to synthesize bone replacement materials for clinical applications featuring physiological tolerance, biocompatibility and long term stability have had only relative success till now. Bioceramics was introduced to orthopedics as bone substituted materials during the 1960s. They have high compressive strength and hardness. In addition, they are highly biocompatible and tissue responsive. According to their tissue response, they can be categorized into three types; (i) bioinert (e.g. alumina and zirconia) ${ }^{[7]}$ (ii) bioactive (e.g. HA and bioglass) ${ }^{[8]}$ and (iii) bioresorbable (tri-calcium phosphate (TCP)). Therefore, among all the calcium phosphates only HAp and TCP are being used as bioceramics.

Sr-HAp and Ba-HAp are also biologically active but are less frequently employed as bone substituted materials. Strontium apatite-cement is used as bone replacement, bone fillings, bone adhesives and for the treatment of osteoporosis ${ }^{[9]}$. Addition of barium apatite to the bone cement provides radio opacity to the bone cement. However, there are limited data available on the synthesis of fine particles of strontium and barium apatites ${ }^{[10-13]}$.

A number of novel processing routes have been developed for preparing fine HAp powders that include solid state reaction, wet chemical method, sol-gel route, hydrothermal reaction, microwave heating, emulsion and microemulsion routes and mechanochemical syntheses. Table 1 lists different methods for the synthesis of HAps and the particle characteristics. Here, we report our results on the precipitation of alkalineearth phosphate $\mathrm{A}_{10}\left(\mathrm{PO}_{4}\right)_{6}(\mathrm{OH})_{2}$ where $\mathrm{A}=\mathrm{Ca}, \mathrm{Sr}$ and $\mathrm{Ba}$ starting from same reactants but treated under different condition: microwave, hydrothermal and reverse micellar route. We have discussed in detail their synthetic condition and a comparison of their morphology and size.

Corresponding Author: A. Ramanan, Department of Chemistry, Indian Institute of Technology Delhi, India 
Table 1: Method for synthesis of HAp.

\begin{tabular}{lll}
\hline Methods & General Remarks & Reference \\
\hline Solid state & Inhomogeneous, large grain size (micro to nano), & {$[14]$} \\
Wet chemical & Irregular shapes, reaction condition: $900-1300^{\circ} \mathrm{C}$ & {$[15,16]$} \\
Precipitation & $\begin{array}{l}\text { Homogeneous, nanograin size, low crystallinity, } \\
\text { reaction condition: room temperature to } 100^{\circ} \mathrm{C}\end{array}$ & {$[17]$} \\
Hydrothermal & $\begin{array}{l}\text { Homogeneous, ultra-fine particles, low crystallinity, } \\
\text { reaction condition: room temperature to } 200^{\circ} \mathrm{C}\end{array}$ & {$[18]$} \\
Mechanochemical & Homogeneous, fine crystals, high temperature and & {$[19]$} \\
hH shock wave & $\begin{array}{l}\text { Easy production, ultra-fine crystals, semi-crystallinity, } \\
\text { room temperature process }\end{array}$ & {$[20]$} \\
Microwave & $\begin{array}{l}\text { High-energy dispersing, nonporous, crystalline } \\
\text { particles with Ca/P molar ratio } 1.43-1.66\end{array}$ & {$[21]$} \\
\hline
\end{tabular}

\section{MATERIALS AND METHOD}

In all the cases, the reactants were pure $\mathrm{Na}_{3} \mathrm{PO}_{4}, \mathrm{CaCl}_{2}$, $\mathrm{SrCl}_{2}$ and $\mathrm{BaCl}_{2}$ obtained from Aldrich. Synthesis of $\mathrm{M}$ HAp $(\mathrm{M}=\mathrm{Ca}, \mathrm{Sr}$ or $\mathrm{Ba})$ powders were carried out by reacting a well-ground mixture of $\mathrm{MCl}_{2}$ and $\mathrm{Na}_{3} \mathrm{PO}_{4}$ in the molar ratio $1.67: 1$ using the following synthetic strategies.

The first method was based on the solid state metathethic reaction (SSM) wherein the mixture was heated in a home-made microwave oven $(\sim 800 \mathrm{~W})$ for half an hour. The product was washed with water to remove sodium chloride, which is a by-product of the reaction and dried around $80^{\circ} \mathrm{C}^{[22,23]}$.

In the second method, a similar mixture of identical composition was taken in $25 \mathrm{ml}$ deionized water and transferred to a $30 \mathrm{ml}$ Teflon lined acid digestion reactor (degree of filling $\sim 80 \%$ ) and heated at $150^{\circ} \mathrm{C}$ for two days. The $\mathrm{pH}$ of the reaction medium remained in between 10-11 before and after the hydrothermal treatment. No additional reagent was used to adjust the $\mathrm{pH}$ of the reaction medium. The initial $\mathrm{pH}$ between 1011 was attained due to aqueous $\mathrm{Na}_{3} \mathrm{PO}_{4}$. The product was washed with distilled water and dried at $80^{\circ} \mathrm{C}^{[24]}$.

The third method involved synthesis of M-HAp through microemulsion technique. Two microemulsions (1 and 2) were prepared as described below. Micro-emulsion 1 was made of from cetyltrimethylammonium bromide (CTAB) as surfactant, n-butanol as co-surfactant, isooctane as hydrocarbon phase and $0.167 \mathrm{M} \mathrm{CaCl}_{2}$ as the aqueous phase. Micro-emulsion 2 comprised of the same constituents as above except for that $0.1 \mathrm{M}$ $\mathrm{Na}_{3} \mathrm{PO}_{4}$ was used instead of $\mathrm{CaCl}_{2}$ as the aqueous phase. The weight fractions of various constituents were as follows: $16.76 \%$ of CTAB, $13.90 \%$ of $n-$ butanol, $59.29 \%$ of isooctane, and $10.05 \%$ of the aqueous phase. These two micro-emulsions were mixed slowly and stirred for $24 \mathrm{hr}$ on a magnetic stirrer. The colloidal solids were isolated by centrifugation followed by washing with 1:1 mixture of methanol and chloroform and drying at $80^{\circ} \mathrm{C}$ in an oven.

Synthesis of Sr-HAp and Ba-HAp nanoparticles were carried out by the above method except that microemulsion 1 composed of $0.167 \mathrm{M} \mathrm{SrCl}_{2}$ and $0.167 \mathrm{M}$ $\mathrm{BaCl}_{2}$ respectively and micro-emulsion 2 composed of $0.1 \mathrm{M} \mathrm{Na}_{3} \mathrm{PO}_{4}$ as the aqueous phase. ${ }^{25}$
The materials were characterized using powder X-ray diffraction (XRD), Fourier Transform Infrared Spectroscopy (FTIR), Thermogravimetric analysis (TGA), ${ }^{31} \mathrm{P}$ solid state NMR, scanning electron microscopy (SEM) and transmission electron microscopy (TEM).

\section{RESULTS AND DISCUSSION}

In this work, we have investigated the formation of monophasic M-HAp $(\mathrm{M}=\mathrm{Ca}, \mathrm{Sr}$ and $\mathrm{Ba})$ via three different routes employing same reactants $\left(\mathrm{Na}_{3} \mathrm{PO}_{4}\right.$ and $\left.\mathrm{MCl}_{2}\right)$ in the same molar ratio. These reactants were chosen to exploit the metathetic pathway through which the reaction occurred. Metathetic pathway requires minimum reagents, assures faster reactivity and enables better homogeneity. In addition, use of aqueous $\mathrm{Na}_{3} \mathrm{PO}_{4}$ (in hydrothermal and reverse micellar route) results in alkaline $\mathrm{pH}$ (10-11) suitable for the crystallization of M-HAp thereby eliminating the need of external agents for pî̀ adjustment. The formation of singie phasic MHAp was established by XRD analysis (Fig. 1).

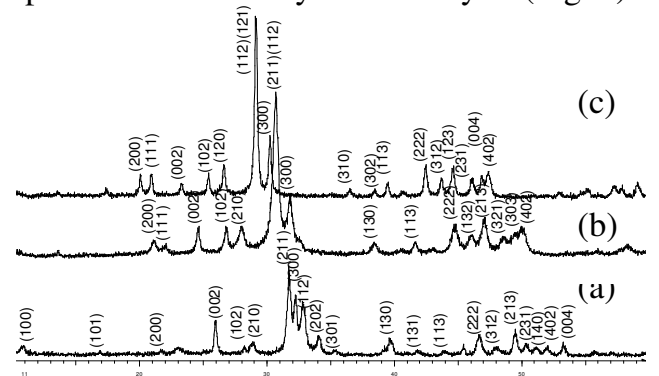

(I)

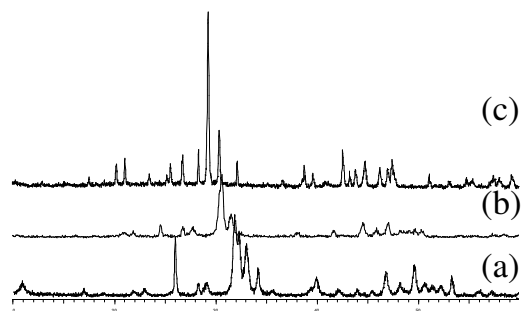

Fig 1. Powder XRD patterns of (a) Ca-HAp, (b) Sr-HAp and (c) Ba-HAp synthesiled via ( I ) Microwave ( II ) hydrothermal methods.

All the reactions resulted in the formation of single phasic M-HAp except Ba-HAp prepared via reverse micellar route (Fig. 2). 

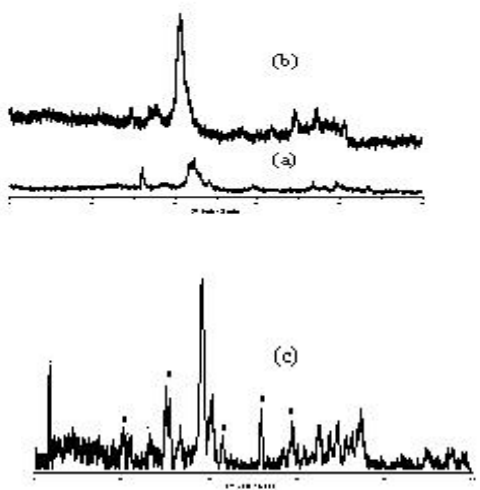

Fig 2. Powder XRD patterns of (a) Ca-HAp, (b) SrHAp and (c) Ba-HAp synthesized via reverse micellar route. * corresponds to peak of $\mathrm{Ba}\left(\mathrm{H}_{2} \mathrm{PO}_{4}\right)$ and 0 corresponds to peak of $\mathrm{BaHPO}_{4}$

However, the crystallinity of the phases obtained in this method were poorer (broader reflections in Fig. 2 in comparison to sharper and intense reflections in Fig. 1). In all the cases, SEM revealed submicron size particles in agglomerated form. Fig 3 shows SEM images of CaHAp synthesized by different routes.



(a)

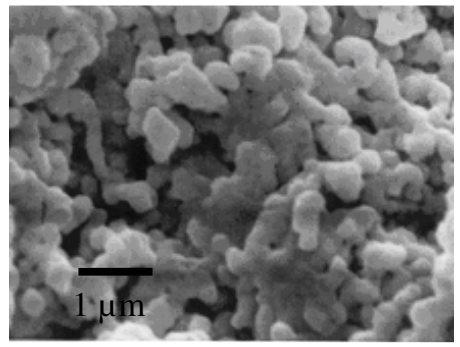

(b)

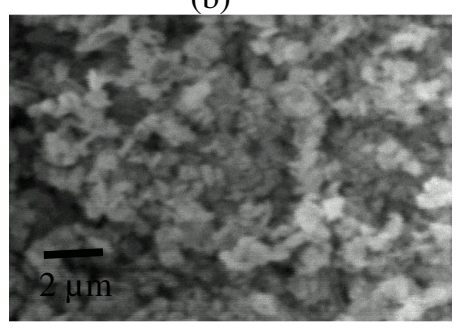

Fig. 3. SEM images of Ca-HAp synthesized by (a) Microwave, (b) hydrothermal, (c) reverse micellar route.
As expected, agglomeration was less in the case of reverse micellar system (Fig.3c) possibly due to the presence of surfactants in the precipitating medium. In Fig 4 we have shown TEM images of Ca-HAp samples obtained under three different methods.

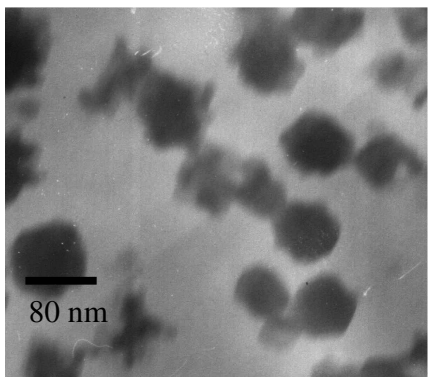

(a)

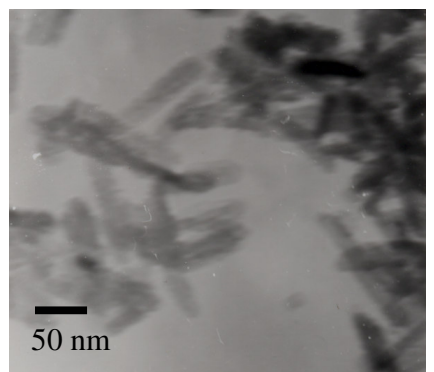

(b)

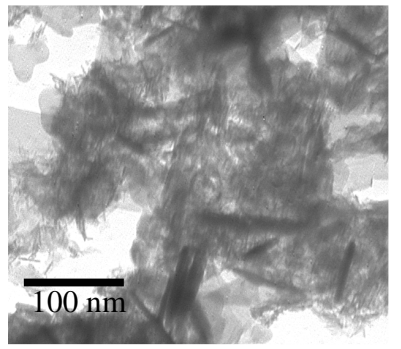

(c)

Fig.. 4. TEM images of Ca-HAp synthesized by (a) microwave, (b) hydrothermal, (c) reverse micellar route.

Ca-HAp particles obtained by SSM route were smaller $(<80 \mathrm{~nm})$ but with irregular shape. Under hydrothermal condition, the particles were better grown, rod-shaped and well dispersed. Unlike these two methods, in reverse micellar route, precipitation occurred in the nanoreactors and the particles were smaller but poorly dispersed. TEM of M-HAp obtained from reverse micellar route clearly shows the presence of uniform naorods; however it is better dispersed in the case of SrHAp (Fig. 5a). In comparison to SSM and reverse micellar route, hydrothermal condition resulted in the growth of larger size nanorods of Sr-HAp and Ba-HAp with high aspect ratio (Figs. 5b and 5c). 


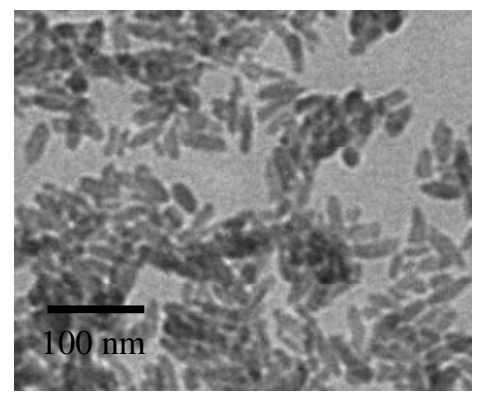

(a)

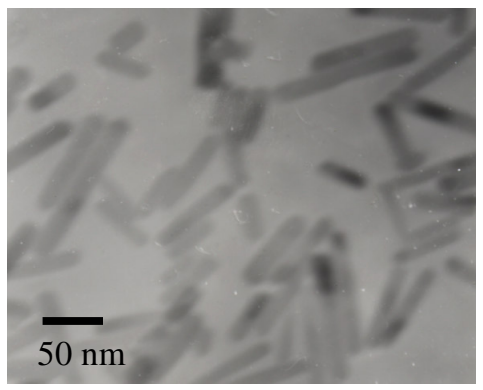

(b)

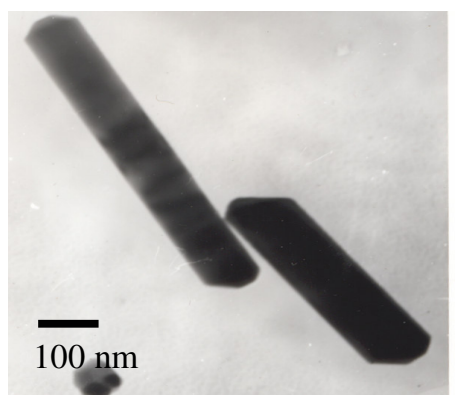

(c)

Fig. 5. TEM images of Sr-HAp prepared by (a) reverse micellar methods and (b) hydrothermal. TEM image of Ba-HAp synthesised by hydrothermal is shown in (c).

Recently, it was recognized that inorganic oxides could be synthesized through metathesis reaction ${ }^{26}$, Our experiments were the first to demonstrate the formation of biomaterials such as M-HAp by this route. The high lattice energy of $\mathrm{NaCl}$ byproduct favors the formation of the thermodynamically stable M-HAp. Also, NaCl could be readily washed off with water. Though formation of M-HAps by SSM route occurred in short duration, it led to fine particles with extensive agglomeration. On the other hand, the metathetic reaction via hydrothermal route between $\mathrm{Na}_{3} \mathrm{PO}_{4}$ and $\mathrm{MCl}_{2}$ in aqueous solution resulted in better crystallinity with nanorods $(10 \mathrm{~nm}$ in diameter and $200 \mathrm{~nm}$ in length) as major products; however bulk preparation again suffered from extensive agglomeration (Figs. 3 and 4).
In reverse micellar method, precipitation occurred in nano bottles with the surface made of surfactant molecules. Here, the reaction of calcium and strontium ions with phosphate ions produced nanorods of $\mathrm{Ca}-$ HAp and Sr-HAp. However, attempts to prepare BaHAp resulted in a mixed-phase containing Ba-HAp, $\mathrm{Ba}\left(\mathrm{H}_{2} \mathrm{PO}_{4}\right)_{2}$ and $\left.\mathrm{Ba}-\mathrm{HPO}_{4}\right)$. The occurrence of varying size and growth characteristics of M-HAp particles can be explained on the basis of the reactivity of $\mathrm{MCl}_{2}$ with $\mathrm{Na}_{3} \mathrm{PO}_{4}$. We opted for these reagents as metathetic nature of the reaction provided an elegant path to obtain nanocrystalline M-HAp. High lattice energy of $\mathrm{NaCl}$ is the principal driving force for the reaction. However, the increasing ionicity from $\mathrm{CaCl}_{2}, \mathrm{SrCl}_{2}$ to $\mathrm{BaCl}_{2}$ (increasing lattice energy) decreases the difference between overall energy changes. This is directly reflected in the metathetic reactivity. In the first method, the reaction proceeded through a solid state route whereas in the other two cases, precipitation led to the formation of products. In the SSM route, composition was controlled by the molar ratio of the reactants whereas in the precipitation method $\mathrm{pH}$ is essentially responsible for phase purity. Reaction of $\mathrm{CaCl}_{2}$ with $\mathrm{Na}_{3} \mathrm{PO}_{4}$ readily occurred even at room temperature (though less crystalline) by mechanical grinding in agate mortar while reaction with $\mathrm{SrCl}_{2}$ and $\mathrm{BaCl}_{2}$ required acceleration through microwave irradiation. The well-grown nanocrystals in the case of $\mathrm{Sr}$ and $\mathrm{Ba}$ in comparison to $\mathrm{Ca}$ are due to slow reactivity of these salts with sodium phosphate either in the solid state or in solution. In particular, the reaction of barium ions with phosphate anions is very sluggish and takes longer time during which $\mathrm{PO}_{4}{ }^{3-}(\mathrm{aq})$ undergoes equilibration with water to produce substantial amounts of $\mathrm{H}_{2} \mathrm{PO}_{4}{ }^{2-}$ and $\mathrm{HPO}_{4}{ }^{2-}$ species and hence different phases especially in the reverse micellar route. Here, not only the reaction occurs at room temperature but also the solvent is a mixture of aqueous and nonaqueous which further affects the solubility of the various competing phosphate anions. Synthesis of alkaline-earth hydroxyapatite was readily achieved by choosing a metathetic route. As expected, the particles were irregular in shape and extensively agglomerated in SSM route due to fast reaction. However, controlled nucleation took place under hydrothermal condition (moderate temperature and higher water vapour pressure) resulting in well-developed nano M-HAp crystals with high aspect ratio. The high $\mathrm{pH}$ in aqueous medium also assists the growth of M-HAp; the size is more apparent in $\mathrm{Ba}$. In the reverse-micellar route, the presence of a surfactant and less polar medium (organic and inorganic) resulted in lesser agglomeration and reduced the size of the particles. Unlike hydrothermal method, here precipitation occurred at room temperature in mixed solvent leading to the domination of competitive phosphate phases. Recently, we successfully employed Ca-HAp nanorods as precursors (prepared by hydrothermal route) for processing into polycaprolactone based nanocomposite. 


\section{CONCLUSIONS}

Unlike the previously reported methods for the preparation of M-HAp, use of sodium phosphate and alkaline-earth chloride as reactants in chemical routes such as microwave, hydrothermal reaction as well as reverse micellar route, invariably resulted in better reactivity and controlled growth of nano-sized M-HAp particles. Our results indicate that the metathetic reactivity of M-HAp is influenced by the lattice energy of the alkaline-earth halides. Slower reaction leads to better grown nanocrystalline phases.

\section{ACKNOWLEDGEMENTS}

PP acknowledges CSIR for a fellowship. AR acknowledges DST-IRHPA for a powder X-Ray diffractometer to the Department of Chemistry, IIT, Delhi. ARR acknowledges ICMR for financial assistance.

\section{REFERENCES}

1. Boyle, W. J., W. S. Simonet and D. L. Lacey, 2003. Osteoclast differentiation and activation. Nature, 423: 337-342.

2. Lakes, R., 1993. Materials with structural hierarchy. Nature, 361: 511-515.

3. Hartgerink, J. D., E. Beniash and S. I. Stupp, 2001. Self-assembly and mineralization of peptide-amphiphile nanofibers. Science, 294: 1684-1688.

4. Currey, J. D., 2002. Bones: structure and mechanics. New Jersey: Princeton University Press.

5. Lowenstam, H. A. and S. Weiner, 1989. On biomineralization. New York: Oxford University Press.

6. McConnell, D., 1962. The crystal structure of bone. Clin. Orthop. Relat. Res., 23: 253-268.

7. Murugan, R. and S. Ramakrishna, 2004. Nanostructured biomaterials. In: Nalwa HS, editor. Encyclopedia of nanoscience and nanotechnology, vol.7. California: American Scientific Publishers. 595-613.

8. Hench, L. L., 1998. Bioceramics. J. Am. Ceram. Soc., 81: 1705-1728.

9. Robert, W., 2003. Strontium-apatite-cementpreparations and the use as bone cement. PCT Int. Appl., 25.

10. Aizawa, M., S.F. Howell and K. Itatani, 1999. Characterization of strontium apatite powders prepared by ultrasonic spray-pyrolysis technique, J. Cer. Soc. Japan., 107: 1007-1011.

11. Ioku, K., Y. Eguchi, H. Fujimori, S. Goto, W. Suchanek and M. Yoshimura, 1999. Porous materials of calcium hydroxyapatite and strontium hydroxyapatite prepared hydrothermally, Phos. Res. Bull., 9: 11-16.

12. Correig, T. M., E. Cardellach, M. A. Cuevas, M. Font-Altaba, 1975. Synthesis of barium apatite by a solid-state reaction. Acta Geol. Hisp., 10: 161-166.
13. Aizawa, M., T. Hanazawa, K. Itatani, F. S. Howell and A. Kishioka, Characterization of various apatites powders prepared by ultrasonic spray-pyrolysis technique. Phos. Res. Bull., 6: 217-220.

14. Ota, Y. and T. Iwashita, 1998. Novel preparation method of hydroxyapatite fibers. J. Am. Ceram. Soc., 81: 1665-1668.

15. Murugan, R. and S. Ramakrishna, 2005. Aqueous mediated synthesis of bioresorbable nanocrystalline hydroxyapatite. J. Cryst. Growth, 274: 209-213.

16. Murugan, R. and S. Ramakrishna, 2004. Bioresorbable composite bone paste using polysaccharide based nano-hydroxyapatite. Biomaterials, 25:3829-3835.

17. Zhang, S. and K. E. Consalves, 1997. Preparation and characterization of thermally stable nanohydroxyapatite. J. Mater. Sci. Mater. Med., 8: 25-28.

18. Ioku, K., S. Yamauchi, H. Fujimori, S. Goto and M. Yoshimura, 2002. Hydrothermal preparation of fibrous apatite and apatite sheet. Solid State Ionics, 151: 147-150.

19. Nakamura, S., T. Tsobe and M. Senna. 2001. Hydroxyapatite nano sol prepared via a mechanochemical route. J. Nanopart. Res., 3: 57-61.

20. Koumoulidis, G. C., T. C. Vaimakis, A. T. Sdoukos, N. K. Boukos and C. C. Trapalis, 2001. Preparation of hydroxyapatite lath-like particles using high-speed dispersing equipment. J. Am. Ceram. Soc., 84: 12031208.

21. Yang, Y. and J. L. Ong, 2002. Rapid sintering of hydroxyapatite by microwave processing. J. Mater. Sci. Lett., 21: 67-69.

22. Parhi, P., A. Ramanan and A. R. Ray, 2006. Synthesis of nano-sized alkaline-earth hydroxyapatites through microwave assisted metathesis route. Mater. Lett., 60: 218-221.

23. Parhi, P., A. Ramanan and A. R. Ray, 2004. A convenient route for the synthesis of hydroxyapatite through a novel microwavemediated metathesis reaction. Mater. Lett., 58: 3610-3612.

24. Parhi, P., A. Ramanan and A. R. Ray, 2006. Hydrothermal Synthesis of nanocrystalline powders of alkaline-earth hydroxyapatites, $\mathrm{A}_{10}\left(\mathrm{PO}_{4}\right)_{6}(\mathrm{OH})_{2}(\mathrm{~A}=\mathrm{Ca}, \mathrm{Sr}$ and $\mathrm{Ba}) . \quad \mathrm{J}$. Mater. Sci., 41: 1455-1458.

25. Parhi, P., A. Ramanan and A. R. Ray, 2006. Metathetic reaction in reverse micelles: synthesis of calcium and strontium hydroxyapatites nanorods, (communicated).

26. Gopalakrishnan, J., T. Shivakumar, K. Ramesha, V. Thangadurai, G. N. Subbanna, (2000). Transformations of ruddlesden-popper Oxides to new layered perovskite oxides by metathesis reactions. J. Amer. Chem. Soc. 122(26): 6237-6241. 